\title{
Analysis of genes of tetrahydrofolate-dependent metabolism from cultivated spirochaetes and the gut community of the termite Zootermopsis angusticollis
}

\author{
Tina M. Salmassit and Jared R. Leadbetter \\ Environmental Science and Engineering, M/C 138-78, California Institute of Technology, \\ Pasadena, CA 91125, USA
}

Correspondence

Jared R. Leadbetter

jleadbetter@caltech.edu

\begin{abstract}
The hindguts of wood-feeding termites are the sites of intense, $\mathrm{CO}_{2}$-reductive acetogenesis. This activity profoundly influences host nutrition and methane emissions. Homoacetogens previously isolated from diverse termites comprised novel taxa belonging to two distinct bacterial phyla, Firmicutes and Spirochaetes. Little else is known about either the diversity or abundance of homoacetogenic species present in any given termite or the genetic details underlying $\mathrm{CO}_{2}$-reductive acetogenesis by Spirochaetes. A key enzyme of $\mathrm{CO}_{2}$-reductive acetogenesis is formyltetrahydrofolate synthetase (FTHFS). A previously designed primer set was used to amplify FTHFS genes from three isolated termite-gut spirochaetes. Sequencing DNA flanking the FTHFS gene of Treponema strain ZAS-2 revealed genes encoding two acetogenesis-related enzymes, methenyltetrahydrofolate cyclohydrolase and methylenetetrahydrofolate dehydrogenase. Although termite-gut spirochaetes are only distantly related to clostridia at the ribosomal level, their tetrahydrofolate-dependent enzymes appear to be closely related. In contrast, homologous proteins identified in the non-homoacetogenic oral spirochaete Treponema denticola were only distantly related to those from clostridia and the termite-gut treponemes. Having demonstrated their utility with spirochaete pure cultures, the FTHFS primers were used to construct a 91-clone library from the termite-gut community DNA. From this, 19 DNA and eight amino acid FTHFS types were identified. Over $75 \%$ of the retrieved clones formed a novel, coherent cluster with the FTHFS homologues obtained from the termite-gut treponemes. Thus, FTHFS gene diversity in the gut of the termite Zootermopsis angusticollis appears to be dominated by spirochaetes. The homoacetogenic capacity of termite-gut spirochaetes may have been acquired via lateral gene transfer from clostridia.
\end{abstract}

Received 14 March 2003

Revised 13 May 2003

Accepted 28 May 2003 high as $2-5 \mu \mathrm{mol} \mathrm{CO}_{2}$-derived acetate (g fresh weight termite $)^{-1} h^{-1}$ have been observed for at least a half dozen wood-feeding termite species (Brauman et al., 1992). No other environment has been demonstrated to catalyse $\mathrm{CO}_{2}$-reductive acetogenesis at such high rates. Gut-derived acetate serves to fuel up to $100 \%$ of the energy metabolism of termites, and it has been estimated that $\mathrm{CO}_{2}$ reduction can generate up to one-third of this key nutrient for the insect (Breznak \& Switzer, 1986; Odelson \& Breznak, 1983). Thus, homoacetogens play an important role in the nutrition of their insect hosts.

In many termite species, bacterial homoacetogens outprocess methanoarchaea for reductant, most notably $\mathrm{H}_{2}$ (Brauman et al., 1992). As a result, termites emit less $\mathrm{CH}_{4}$ than might be expected if their fermentation pattern were similar to that observed in ruminants, and the basis for this is not well understood. Termites are as intriguing for 
the $\mathrm{CH}_{4}$ that they do not emit, as much as for the $\mathrm{CH}_{4}$ that they do.

Because of the influence of acetogenesis on termite nutrition and the production of greenhouse gases, there has been considerable interest in learning more about the gut microbiota accounting for this activity (Breznak, 1994). Four homoacetogenic, spore-forming Firmicutes have previously been isolated from four different termite species and described as novel taxa (Boga et al., 2003; Breznak et al., 1988; Kane \& Breznak, 1991; Kane et al., 1991). Two homoacetogenic spirochaetes have been isolated from a fifth termite species (Leadbetter et al., 1999). The latter strains, Treponema ZAS-1 and Treponema ZAS-2, represented the first examples of spirochaetes isolated from any termitegut environment, as well as the first demonstration of lithotrophic metabolism (much less $\mathrm{CO}_{2}$-reductive acetogenesis) in this phylum-level group of bacteria. The isolates exhibited enzymic activities associated with the WoodLjungdahl pathway used by homoacetogenic Firmicutes (Leadbetter et al., 1999), a pathway also used in the oxidative energy metabolism and autotrophic anabolism of diverse archaea and sulfate-reducing bacteria (Drake et al., 1997; Ragsdale, 1997; Wood, 1991). However, the evolutionary history of this pathway in spirochaetes, and its relationships to those from better-studied organisms, is not currently known. The actual contribution to gut acetogenesis by any of the aforementioned isolates and the full diversity of homoacetogens present and active in any single termite species are also poorly understood. It has proven tempting to speculate that spirochaete species may dominate acetogenesis in the guts of termites (Leadbetter et al., 1999), especially since spirochaetes are among the most numerous microbiota present in the guts of virtually all termite species, representing as many as $50 \%$ of all prokaryotic cells (Breznak \& Leadbetter, 2002; Lilburn et al., 1999; Paster et al., 1996). However, at least $50 \%$ of the prokaryotic cells found in termite guts are not spirochaetes and not all Zootermopsis-gut spirochaetes are $\mathrm{CO}_{2}$-reducing acetogens. One strain isolated from this environment, Treponema ZAS-9, does not display this mode of metabolism (Breznak \& Leadbetter, 2002; Lilburn et al., 2001).

We have adopted the use of a functional-gene approach employing degenerate, oligonucleotide primers developed to amplify portions of genes encoding a key enzyme of the $\mathrm{CO}_{2}$-reductive acetogenesis pathway: formyltetrahydrofolate synthetase (FTHFS) (Leaphart \& Lovell, 2001; Leaphart et al., 2003). These primers were successfully applied to three termite-gut spirochaete isolates. The sequences for FTHFS partial genes for two of these spirochaetes have also been recently reported by others (Leaphart et al., 2003). Here, we detail our analysis of a sizeable collection of FTHFS genes derived from spirochaetal isolates and the gut community of the termite Zootermopsis, as well as two putative acetogenesis-related genes flanking the FTHFS gene in Treponema strain ZAS-2.

\section{METHODS}

Termites and strains. Specimens of Zootermopsis angusticollis, the Pacific dampwood termite, were collected from a rotted Pinus ponderosa (Pacific ponderosa pine) stump located on the north face of the summit of Mount Wilson in the San Gabriel Mountains of Southern California. Specimens were healthily maintained in the laboratory in polypropylene trays containing pieces of the stump material and were used within a month of their collection. Microbial isolates examined in the study [Treponema strains ZAS-1 (DSM 12426), ZAS-2 (DSM 12427) and ZAS-9 (DSM 13862)] and media for their cultivation have been described previously (Breznak \& Leadbetter, 2002; Leadbetter et al., 1999; Lilburn et al., 2001).

DNA extraction. Z. angusticollis-gut community DNA was purified using a modification of a previously described technique (Purdy et al., 1996). DNA was extracted from the entire, intact hindgut of nine uniformly sized worker larvae. Three termite hindguts were combined into each of three $2 \mathrm{ml}$ microcentrifuge tubes containing $0.2 \mathrm{~g}$ of $0.1 \mathrm{~mm}$ Biospec zirconium/silica beads, $700 \mu \mathrm{l}$ of $1 \%$ $(\mathrm{w} / \mathrm{v})$ polyvinylpolypyrrolidone in $120 \mathrm{mM}$ sodium phosphate, $\mathrm{pH} 8 \cdot 0,50 \mu \mathrm{l}$ of $20 \%(\mathrm{w} / \mathrm{v})$ SDS and $500 \mu \mathrm{l}$ TRIS-equilibrated phenol, $\mathrm{pH} 8 \cdot 0$. The samples were disrupted using a Biospec MiniBeadbeater-8 as described previously (Purdy et al., 1996). The reaction mixtures were pooled and applied to hydroxyapatitecontaining microcentrifuge spin columns (Bio-Rad). Sample application, washing and elution steps were performed by gently spinning the column for $<10 \mathrm{~s}$ in a fixed-angle bench-top microcentrifuge. DNA was extracted from the treponemal isolates using the DNeasy extraction kit (Qiagen). DNA samples were stored at $-20^{\circ} \mathrm{C}$ until used.

PCR amplification, cloning and RFLP analysis. FTHFS homologues were amplified from DNA templates prepared from cultivated spirochaete strains and termite-gut contents using the primers and PCR conditions exactly as described by others previously (Leaphart \& Lovell, 2001), except that Roche Taq polymerase and reagents were used. Reactions yielded the expected $\sim 1 \cdot 1 \mathrm{~kb}$ gene fragments as products. When community DNA was used as template, reaction products of various other sizes were also observed. PCR products generated from genomic templates from isolated strains were cloned directly using the Topo TA cloning kit (Invitrogen); products amplified from gut template were similarly cloned after purification of the desired-size band using the QIAEX II Gel Extraction Kit (Qiagen). Using an adaptation of a previously described method ( $\mathrm{Ng}$ et al., 1996), the community-derived clone library was characterized via RFLP analyses after double digestion with MspI and HinP1I following the manufacturer's recommendations (New England Biolabs). RFLPs were visualized via gel electrophoresis using $2 \cdot 5 \%$ $(\mathrm{w} / \mathrm{v})$ low-melting-point agarose and analysed manually.

Inverse PCR generation of Treponema ZAS-2 genomic fragment. A synopsis of this technique has been described previously (Raponi et al., 2000). Genomic DNA was digested with NcoI according to the manufacturer's guidelines (New England Biolabs). The digested-DNA fragments were circularized using T4 DNA ligase. The ligation mixture was purified using the QIAEX II Gel Extraction Kit (Qiagen). Five microlitres of the purified ligation mixture was used as template in $20 \mu \mathrm{l}$ reactions using the Failsafe PCR System and PCR PreMix Selection Kit (Epicenter). The internal FTHFS 'out' primers employed were 5'-GGCCGAAGAAGTCGTAAAAC-3', designed to amplify the downstream gene sequence, and $5^{\prime}$ CCAGGTTCGGTTTCAGGGC- ${ }^{\prime}$, designed to amplify the upstream gene sequence. The amplification conditions used were initial denaturation at $94^{\circ} \mathrm{C}$ for $3 \mathrm{~min}$, followed by 30 cycles of denaturation at $94{ }^{\circ} \mathrm{C}$ for $30 \mathrm{~s}$, annealing at $55^{\circ} \mathrm{C}$ for $1 \mathrm{~min}$ and extension at 
$72{ }^{\circ} \mathrm{C}$ for $6 \mathrm{~min}$, with a final extension at $72{ }^{\circ} \mathrm{C}$ for $10 \mathrm{~min}$. Products were RFLP-sorted and cloned as outlined above.

Nucleotide sequencing and analysis. Sequencing was performed at the DNA Sequencing Core Facility at the Beckman Institute of Caltech using the dideoxy chain termination method, Sequenase (United States Biochemical) and a Perkin-Elmer ABI 373A automated sequencer. Sequence reads were assembled and edited using SEQUENCHER software for Windows (Genecodes). Multiple sequence alignments, translations and phylogenetic analyses were performed using the ARB freeware package (http://www.arb-home.de/) running within the Linux environment. FTHFS trees were constructed using PUZZLE-MAP 5.0 maximum-likelihood analyses (Schmidt et al., 2002). For analyses of aligned protein sequences, 10000 puzzlingsteps were employed using the WAG model for substitution-rate frequencies and uniform models for rate heterogeneity to construct the input matrices (Goldman \& Whelan, 2000). For analysis of FTHFS clones at the DNA sequence level, the Schöniger \& von Haeseler substitution and uniform rate heterogeneity models were employed (Schöniger \& von Haeseler, 1999). Transversion parameters and doublet frequencies were estimated from the dataset by the software. Phylogenetic-tree layout-editing was performed using TREEVIEW 1.6.6 for Windows (Page, 1996). Collector's curve analyses were used to estimate the completeness of the clone inventory and were performed using ANALYTIC RAREFACTION 1.3, written and made freely available by Steven M. Holland (http://www.uga.edu/ strata/ software/), and ESTIMATES $6.01 \mathrm{~b} 1$, written and made freely available by Robert K. Colwell (http://viceroy.eeb.uconn.edu/EstimateS).

Nucleotide accession numbers. The GenBank accession numbers for the sequences generated in this study and other FTHFS sequences used in the construction of Fig. 2 are: Moorella thermoacetica, J02911; Horse Manure Clone H 1, AF295711; Horse Manure Clone H 2, AF295715; Horse Manure Clone H 4, AF295714; Horse Manure Clone H 5, AF295713; Spartina Clone SR 10, AF295723; Acetobacterium woodii, AF295701; Clostridium aceticum, AF295705; Clostridium magnum, AF295703; Eubacterium limosum, AF295706; Proteus vulgaris, AF295710; Ruminococcus productus, AF295707; Sporomusa ovata, AF295708; Sporomusa termitida, AF295709; Thermoanaerobacter kivui, AF295704; Za-gut Clone A, AY162294; Za-gut Clone C, AY162295; Za-gut Clone E, AY162296; Za-gut Clone E2, AY162297; Za-gut Clone F, AY162298; Za-gut Clone F2, AY162299; Za-gut Clone G, AY162300; Za-gut Clone G2, AY162301; $Z a$-gut Clone H, AY162302; Za-gut Clone I, AY162303; Za-gut Clone L, AY162304; Za-gut Clone M, AY162305; Za-gut Clone N, AY162306; Za-gut Clone P, AY162307; Za-gut Clone R, AY162308; Za-gut Clone T, AY162309; Za-gut Clone U, AY162310; Za-gut Clone Y, AY162311; Za-gut Clone Z, AY162312; Treponema strain ZAS-1A, AY162313; Treponema strain ZAS-1B, AY162314; Treponema strain ZAS-2, AY254548; Treponema strain ZAS-9, AY162316. An FTHFS gene was identified in the unpublished genome of Treponema denticola (http://www.hgsc.bcm.tmc.edu/ microbial/Tdenticola/).

The 2371 bp genome contig containing three tetrahydrofolate (THF)dependent enzyme-encoding genes from Treponema strain ZAS-2 has been deposited in GenBank under accession number AY254548. Sequences used in the construction of Fig. 4 were obtained from the NCBI BLAST with microbial genomes (http://www.ncbi.nlm.nih.gov/ sutils/genom_table.cgi?) or DOE Joint Genome Institute (http:// www.jgi.doe.gov/JGI_microbial/html/index.html) from both published and unpublished genome projects (Bao et al., 2002; Casjens et al., 2000; Eisen et al., 2002; Ferretti et al., 2001; Fraser et al., 1998; Goodner et al., 2001; Kapatral et al., 2002; Kuroda et al., 2001; Nolling et al., 2001; Ruepp et al., 2000; Shimizu et al., 2002; White et al., 1999; Wood et al., 2001).

\section{RESULTS}

\section{Amplification and cloning of FTHFS homologues from three cultivated termite-gut treponemes}

Two nearly identical FTHFS homologues were cloned from Treponema strain ZAS-1, and one each was cloned from Treponema strain ZAS-2 and the termite-gut diazotroph Treponema strain ZAS-9. The latter strain is not known to be a homoacetogen (Lilburn et al., 2001). When aligned, the FTHFS homologues from the three termite-gut treponemes shared $82 \cdot 2-88 \cdot 8 \%$ identity with each other at the amino acid level and 77-78\% identity with each other at the DNA level. They shared $74-78 \%$ amino acid identity with the FTHFS homologue identified from the bona fide homoacetogen Clostridium magnum, but were completely dissimilar in DNA sequence, even at the level of $\mathrm{G}+\mathrm{C}$ content $(53 \cdot 3-56 \cdot 4 \mathrm{~mol} \%$ for the spirochaetes, in comparison to $36 \cdot 6 \mathrm{~mol} \%$ for Clostridium magnum). The FTHFS homologues from the three termite-gut treponemes shared less than $50 \%$ amino acid identity with the FTHFS homologue identified via BLAST homology-searching of the unpublished genome sequence of the non-homoacetogenic, human oral treponeme, $T$. denticola. The published genomes of two other spirochaetes, Treponema pallidum and Borrelia burgdorferi, did not contain any obvious FTHFS homologues.

\section{Amplification and cloning of diverse FTHFS homologues from the termite-gut community}

DNA was purified from the gut contents of robust specimens. Amplification reactions using the FTHFS primer set and gut DNA as template successfully yielded products of the anticipated $\sim 1 \cdot 1 \mathrm{~kb}$ size. These were ligated into a cloning vehicle and transformed into Escherichia coli. From this, a library of 91 insert-containing plasmid clones was collected. After sorting of the library by comparing RFLP patterns, 31 clones were targeted for sequencing and further analyses. BLAST analyses confirmed that all of the clones encoded FTHFS homologues. When the 31 sequences were aligned and compared with each other, several were found to share near identity (greater than $99.5 \%$ identity at the DNA level) and were thereafter considered as being representative of the same genotype. From these data, 19 unique clone types were established. Each was assigned a Za-gut epithet (for Zootermopsis angusticollis). After in silico translation of FTHFS genes, the deduced amino acid sequences for the $19 \mathrm{Za}$-gut clone types were aligned and compared. Despite clear differences at the DNA level, many of these were nearly identical in their amino acid sequences. Using $98 \%$ amino acid identity as a cut-off, the clone inventory was collapsed into eight distinct amino acid groups. The rank abundances of the DNA and amino acid types recovered in the 91-clone collection are presented in Fig. 1. At both levels, the recovery of unique clones in the collection appeared to be close to saturation: rarefaction and collector's curve analyses indicated that the 

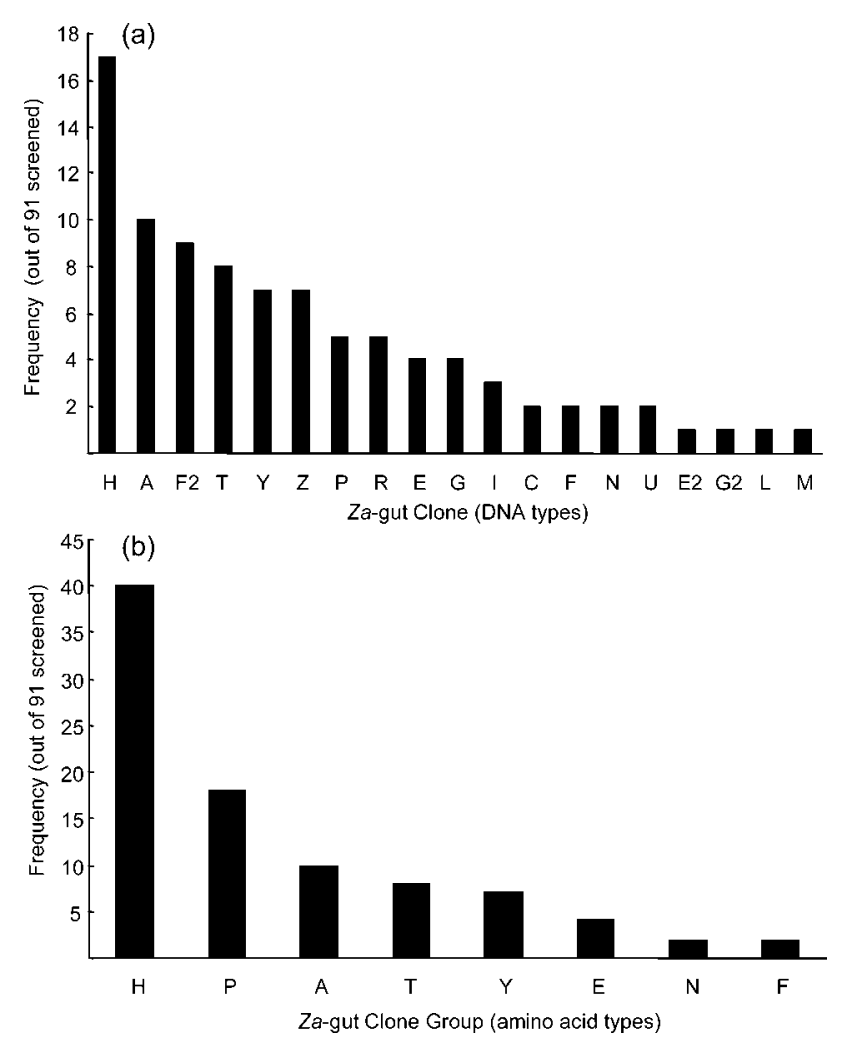

Fig. 1. Rank abundances of recovered FTHFS Za-gut clone types. (a) Frequency at which 19 distinct DNA types were encountered in a 91-clone inventory of FTHFS partial genes amplified from the gut contents of $Z$. angusticollis. Upon translation, the 19 DNA types collapsed into eight distinct amino acid clone groups. (b) Frequency at which the eight distinct amino acid clone groups were encountered in the inventory.

FTHFS inventory was essentially complete using this particular template DNA and primer set. With 'infinite' sampling, rarefaction analysis suggested that no more than 23 DNA and nine amino acid types would have been recovered. Chou and ACE estimators (available within the ESTIMATES program) predicted that no more than 22 DNA and the observed eight amino acid types would have been recovered.

\section{Phylogenetic analysis}

Using distance (not shown), maximum-parsimony (not shown) and maximum-likelihood methods (Fig. 2a), the deduced FTHFS peptides from Treponema strains ZAS-1, ZAS-2 and ZAS-9 clustered within the clostridialhomoacetogen FTHFS homology group previously identified by Lovell and co-workers. Within this cluster of known homoacetogens (Fig. 2a), strain ZAS-9 is the only cultivated organism not known to have this physiology. With the exception of the spirochaetes, which belong to their own unique phylum, all other sequences comprising the Lovell cluster were derived from clostridia, i.e. belonging
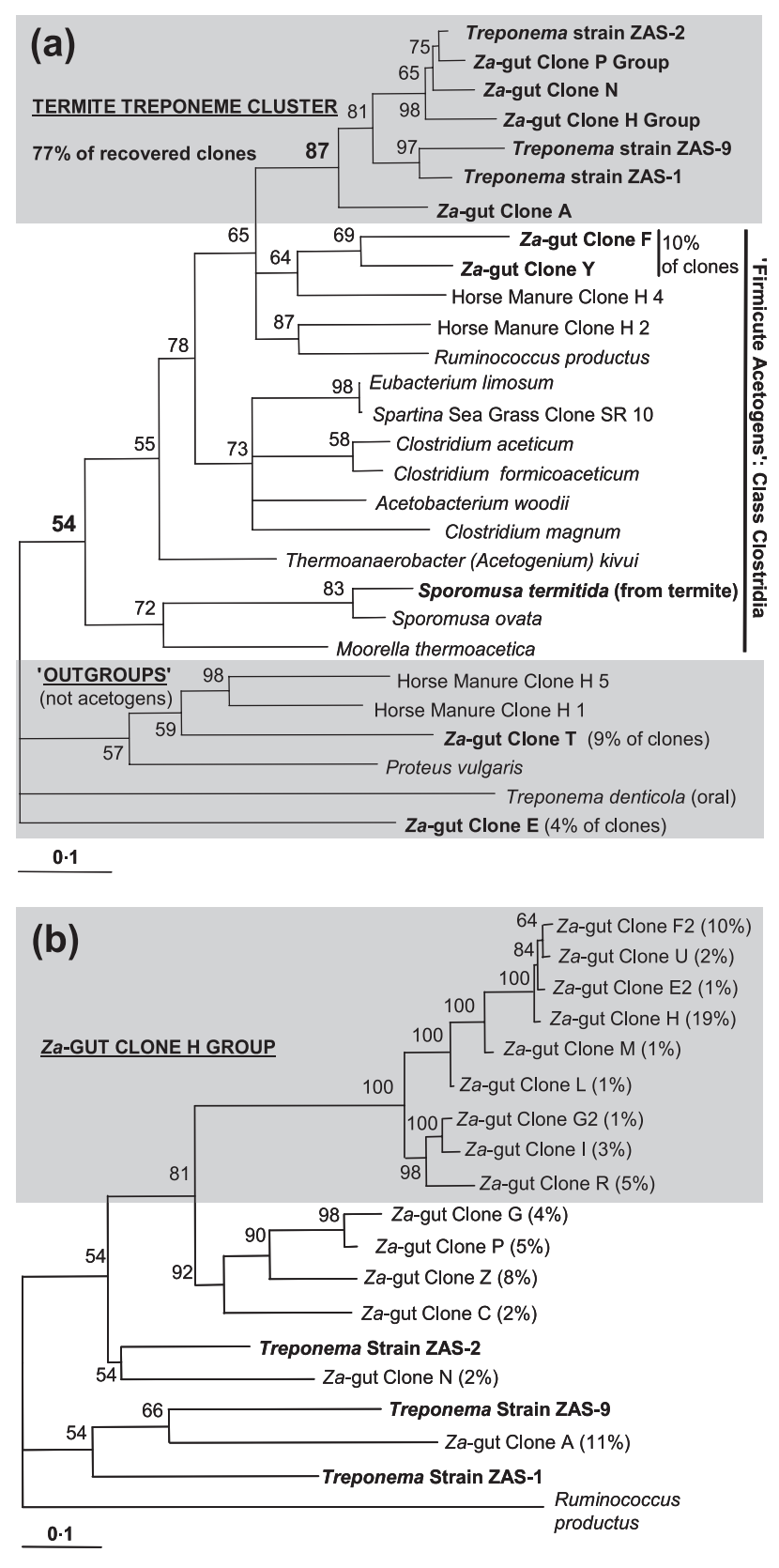

Fig. 2. Phylogenetic position of FTHFS homologues cloned from termite-gut contents, gut isolates or other isolates and environments. Percentages denote frequency of the encounter of a particular clone type or group within the inventory. Numbers are bootstrap values. Bars represent evolutionary distance as 0.1 changes per amino acid or nucleotide position. See Methods for GenBank accession numbers. (a) Maximum-likelihood phylogeny of the deduced FTHFS protein sequences, generated using 323 unambiguously aligned amino acid residue positions. Shaded boxes highlight the termite treponeme cluster (upper) and presumptive or known non-homoacetogens (lower) used to outgroup the unrooted tree. (b) Maximum-likelihood phylogeny of FTHFS genes comprising the termite treponeme cluster, generated using 1062 unambiguously aligned nucleotide positions. The FTHFS gene from $R$. productus was used to outgroup this unrooted tree. Shaded box highlights clones comprising the Za-gut Clone $\mathrm{H}$ group. 
to the phylum Firmicutes, or were derived from not-yetcultivated organisms (Leaphart \& Lovell, 2001).

Over $85 \%$ of the FTHFS homologues recovered from termite-gut contents grouped within the Lovell cluster, and most of these comprised a unique, distinct and wellsupported branch together with the homologues obtained from the treponemal isolates (Fig. 2a). All clone types comprising this 'termite treponeme' cluster shared greater than $80 \%$ amino acid identity with those derived from the treponemal isolates. Of particular note, members of $\mathrm{Za}$ gut Clone $\mathrm{P}$ group and $\mathrm{Za}$-gut Clone $\mathrm{N}$ shared $96 \cdot 3$ and $94.3 \%$ sequence identity, respectively, with the FTHFS homologue identified from Treponema strain ZAS-2. The nine clone types comprising the $\mathrm{H}$ group accounted for $43 \%$ of the clones recovered from gut-community DNA, and shared $\sim 91 \%$ amino acid identity with the FTHFS homologue from strain ZAS-2. Despite close similarities with each other at the amino acid level ( $>98 \%$ identity), clone types comprising the $\mathrm{Za}$-gut Clone $\mathrm{H}$ group were far from identical at the DNA level, thus they appear to represent different microbial strains or species (Fig. 2b). For example, $Z a$-gut Clone F2 and $Z a$-gut Clone R shared only $88 \%$ DNA sequence identity despite sharing $99 \cdot 3 \%$ amino acid identity.

Two Lovell-cluster clone types, $\mathrm{Za}$-gut Clone $\mathrm{F}$ and $\mathrm{Za \text {-gut }}$ Clone $\mathrm{Y}$, showed no affiliation with the termite treponemes (Fig. 2a). These, together with a clone recovered from horse manure DNA, formed a unique radiation and were not highly similar to homologues identified in known homoacetogens. They shared less than $75 \%$ amino acid identity with the FTHFS from $R$. productus. Thus they may represent a novel, not-yet-cultivated microbial lineage. None of the recovered clones evidenced any affiliation with the FTHFS homologue identified in S. termitida, the spore-forming homoacetogen isolated from the gut of the Caribbean wood-feeding, 'higher' termite, Nasutitermes nigriceps. Approximately $15 \%$ of the recovered clones (i.e. Za-gut Clones $\mathrm{T}$ and $\mathrm{E}$ ) did not affiliate with the Lovell cluster and thus may not represent homoacetogens. When our analyses of termite-gut-derived sequences were expanded to include a recently reported, larger collection of FTHFS homologues (Leaphart et al., 2003), none of these aforementioned relationships were meaningfully changed or further resolved. Za-gut Clone E, for example, remained quite remote from any available FTHFS sequences.

\section{Character analysis of FTHFS sequences}

With a few minor exceptions, all FTHFS genes retrieved during the course of this study contained all of the conserved FTHFS polypeptide hallmarks that have previously been noted (Leaphart \& Lovell, 2001; Leaphart et al., 2003). The majority of the FTHFS genes comprising the 'termite treponeme cluster' contained a distinguishing polypeptide character not observed in other FTHFS genes analysed. As many as eight amino acid residues, instead of the more typically encountered two, were found inserted into a region



Fig. 3. Highly variable region found within diverse FTHFS homologues. Shown is a partial amino acid alignment corresponding to a stretch of residues corresponding to Phe222 to Leu241 in the FTHFS enzyme from M. thermoacetica (Lovell et al., 1988; Radfar et al., 2000a, b). Arrows denote $\beta$-sheets evidenced in the crystal structure of the FTHFS from $M$. thermoacetica. The box highlights an eight-residue stretch that is essentially unique to FTHFS homologues cloned from Zootermopsis-gut (Za-gut) Treponema isolates and community DNA. With the exception of those cloned from environmental sources and from Treponema strain ZAS-9, all the listed homologues are from organisms shown to be homoacetogens.

corresponding to Moorella residues Tyr231 and Lys 234 (Fig. 3). Of all other FTHFS homologues analysed, only $R$. productus contained additional residues within this region. This unique region was filtered from alignments used for making phylogenetic comparisons, thus serves as an independent character or 'signpost' providing additional support for the coherency of the termite treponeme radiation.

\section{Inverse PCR-mediated cloning of FTHFS-flanking DNA}

Using sequence from the partial gene, outward-oriented primers were designed to amplify DNA flanking the FTHFS locus in Trepomema strain ZAS-2 (see Methods). The primer set was used in an inverse PCR (iPCR) amplification (see Methods). From this, an 1880 bp genome fragment was amplified, cloned and sequenced. The resultant data, when assembled together with those for the FTHFS partial gene, resulted in $2371 \mathrm{bp}$ of contiguous sequence. This included sufficient data to evaluate the site targeted by Lovell's forward primer, i.e. used to amplify the FTHFS partial gene. The site was identical to a single oligonucleotide ( $5^{\prime}$-TTCACTGGTGATTTCCATGCC- $\left.3^{\prime}\right)$ in the 24 -fold degenerate 'FTHFS forward primer' mixture. No 
other upstream data were revealed, as the majority of the iPCR-generated sequence corresponded to the region immediately downstream from the FTHFS partial gene. This included an unambiguous sequence that contained a single nucleotide mismatch in the binding site $\left(5^{\prime}\right.$ TGCATGGCCAAGACCCAATACAGC-3'; with C in place of the target $G$, previously considered to be invariable) for an oligonucleotide in the 12-fold degenerate 'FTHFS reverse primer' mixture. The contig also included a sequence encoding the remainder of the carboxyl-terminus of the FTHFS homologue.

Downstream from the FTHFS-encoding gene was an obvious ORF. The deduced 209 residue protein shared $50 \%$ amino acid sequence identity with a putative methenyltetrahydrofolate cyclohydrolase (MTHFC) from the unpublished genome of Desulfitobacterium hafniense, a spore-forming member of the class Clostridia. MTHFCs catalyse the enzymic step in acetogenesis following that exerted by FTHFS and are also involved in other THFdependent metabolisms. Because the gene encoding this enzyme was not available from any known homoacetogen, the homologue from strain ZAS-2 was compared with those identified homologues from the D. hafniense and 20 other, mostly unpublished, genome sequences. It clustered within a well-supported branch along with the homologues from D. hafniense, Clostridium botulinum, Clostridium difficile and Carboxydothermus hydrogenoformans, all of which are Firmicutes belonging to the class Clostridia (Fig. 4a). This radiation was distinct from the branch which contained a MTHFC homologue identified from the unpublished genome of the non-acetogenic, human oral treponeme, T. denticola (Fig. 4a). As was the case for FTHFS-encoding genes, the published genomes of two other spirochaetes, T. pallidum and B. burgdorferi, did not contain any obvious MTHFC homologues.

Downstream from the putative MTHFC homologue from Treponema strain ZAS-2 was a partial gene interrupted by the terminus of the iPCR-generated amplification product. The first 102 amino acids of the deduced polypeptide were homologous to the catalytic domains of FolD-type methylenetetrahydrofolate dehydrogenases (MTHFDs), and shared $40 \%$ amino acid identity with that from the unpublished genome of $D$. hafniense. MTHFD catalyses the enzymic step in acetogenesis that follows those exerted by FTHFS and MTHFC and is also involved in other THF-dependent metabolisms in other organisms. As was the case with MTHFC, the gene encoding a MTHFD was not yet available from any known homoacetogen. Accordingly, the ZAS-2 homologue was used in a phylogenetic comparison with those identified in the $D$. hafniense and 10 other, mostly unpublished, genome sequences. The homologue from strain ZAS-2 was the deepest branch on a well-supported cluster comprising the enzyme homologues from D. hafniense, Clostridium botulinum, Clostridium difficile and Carboxydothermus hydrogenoformans (Fig. 4b). FolD homologues identified in the
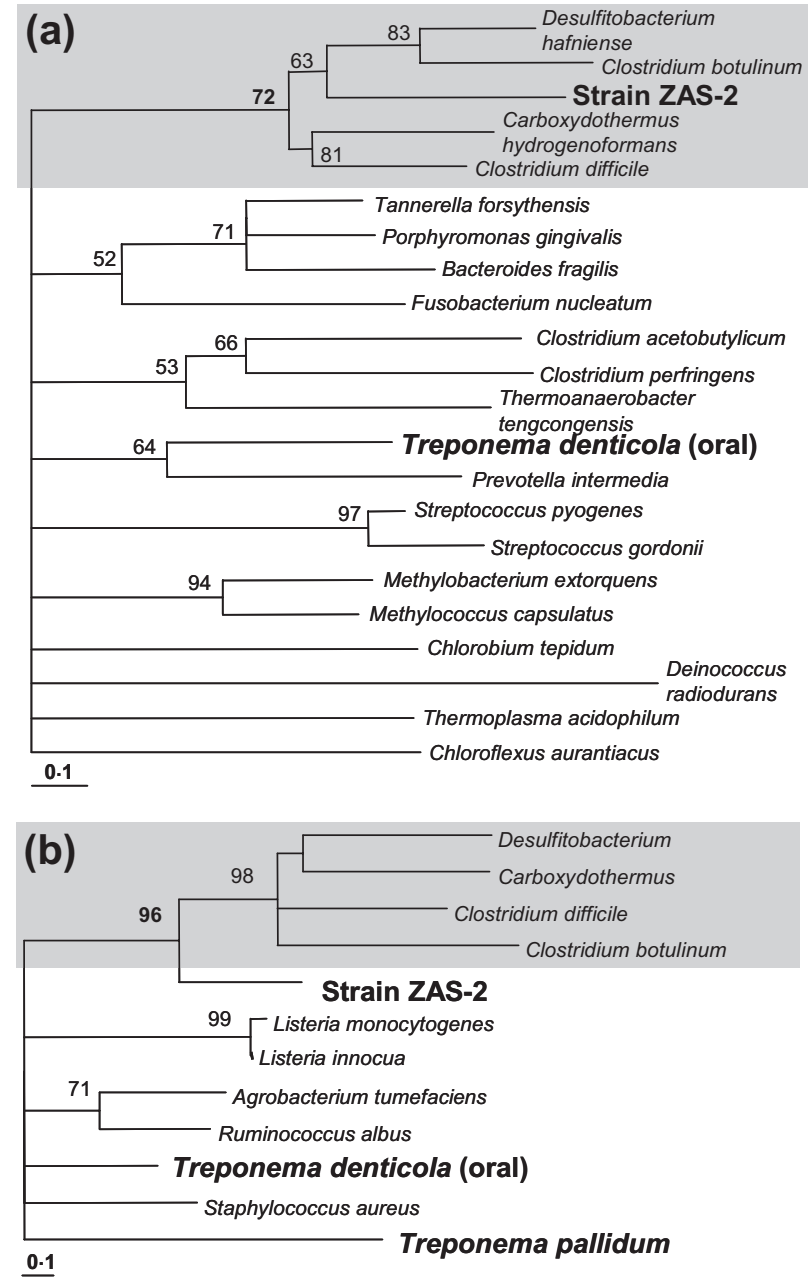

Fig. 4. Phylogenetic position of THF-dependent enzyme homologues cloned from Treponema strain ZAS-2. Homologues of these genes have not yet been cloned from any other homoacetogen. The majority of the homologues used in the analysis were obtained from unpublished genome sequences. Numbers are bootstrap values. Bars represent evolutionary distance as 0.1 changes per amino acid or nucleotide position. (a) Phylogeny of 'MTHFC' homologues. Maximum-likelihood analysis used 174 unambiguously aligned amino acid residue positions of the deduced protein sequences. Shaded box highlights a cluster comprising homologues from Treponema strain ZAS-2 and from four anaerobes belonging to the class Clostridia. (b) Phylogeny of the catalytic region of bifunctional 'FolD-type' and other 'MTHFD' homologues. Maximum-likelihood analysis used 95 unambiguously aligned amino acid residue positions of the deduced protein sequences. Shaded box highlights the cluster formed by the homologues from the same four clostridia comprising the box shaded in (a) (above).

unpublished genome sequences of the non-homoacetogenic oral treponeme $T$. denticola, and published genome of the syphilis spirochaete T. pallidum, did not cluster phylogenetically with Treponema strain ZAS-2 or the four clostridia (Fig. 4b). As was the case for genes encoding 
FTHFS and MTHFC, the published genome of the Lyme disease spirochaete $B$. burgdorferi did not contain an obvious MTHFD homologue. The branching topology did not change significantly with the addition of more than 50 other demonstrated or deduced MTHFDs to the analysis (not shown).

\section{DISCUSSION}

We have successfully used FTHFS primers to amplify genes likely to be involved in the $\mathrm{CO}_{2}$-reductive metabolism of termite-gut spirochaetes and the gut community of $Z$. angusticollis. We have cloned and analysed (1) FTHFS partial genes encoded by Treponema strains ZAS-1, ZAS-2 and ZAS-9, including extended sequence for strain ZAS-2, (2) a substantial collection of diverse FTHFS partial genes obtained from the gut community of $Z$. angusticollis, and (3) loci encoding two putative acetogenesis pathway enzymes from ZAS-2. To our knowledge, this represents the first appraisal of homoacetogen diversity in any known 'hot spot' for this activity, and also the first report on two key acetogenesis-related genes from any of the $\sim 100$ established species of homoacetogens.

Members of the genus Treponema, a genus within the phylum-level assemblage Spirochaetes, are only distantly related to the classical homoacetogens, most of which belong to the phylum Firmicutes. Treponemes are also only distantly related to other homoacetogens, such as Desulfotignum phosphitoxidans, a $\delta$-proteobacterium, and Holophaga foetida, one of the few cultivated representatives of the phylum Acidobacteria (Liesack et al., 1994; Schink et al., 2002). It was somewhat surprising that primers designed to amplify FTHFS genes from clostridia functioned in doing so from termite-gut spirochaetes, an observation that has also been recently reported by others (Leaphart et al., 2003). More surprising, FTHFS homologues from termite-gut Treponema strains clustered phylogenetically with those from homoacetogenic clostridia and not, for example, with that from the non-homoacetogenic oral spirochaete $T$. denticola (Fig. 2a). This pattern was re-encountered during the analysis of each of two other genes likely to encode THF-dependent enzymes identified from Treponema ZAS-2 (Fig. 4a, b). This suggests that THF-dependent enzymes from closely related termite-gut and human-associated treponemes may have distinctly different evolutionary origins. Thus, it can be postulated that the termite-gut spirochaete pathway may have been acquired via lateral gene transfer from a species closely related to $R$. productus. If so, was the event before or after spirochaetes had first become established within the symbiotic, termite-gut communities? The identification of a 'homoacetogen-like' FTHFS gene from the $\mathrm{H}_{2}$-producing isolate Treponema ZAS-9 raises similar intrigue, as this strain is not a homoacetogen (Breznak \& Leadbetter, 2002; Lilburn et al., 2001). Of all the FTHFS genes that comprise Lovell's homoacetogen cluster, to date this is the only example representing a known non-homoacetogen. Might this strain represent a 'snapshot' of a species in evolutionary transition either into or from having $\mathrm{CO}_{2}$-reductive capacities?

Our initial concerns that the FTHFS primer set might be biased towards the overamplification of genes from the homoacetogenic clostridia (for which they had been designed) did not play out. Three-quarters of the clones recovered clustered closely with the spirochaete-derived sequences, several sharing remarkable amino acid similarities with the isolates, for example, 96.3\% similarity shared between $\mathrm{Za}$-gut Clone $\mathrm{P}$ and Treponema ZAS-2 (Fig. 2a). The results of this study establish that there could be as many as 20 strains representing at least two homoacetogenic phyla present in Zootermopsis guts. We are now interested in examining the nature and physical location of the cells encoding these genes, and in determining which types actually dominate FTHFS expression in situ. Are the organisms representing Clone $\mathrm{P}$ and Clone $\mathrm{H}$ truly spirochaetes, are they as abundant and active as the inventory results might suggest, and are they actually homoacetogens? Are the cells representing Clone F and Clone Y actually spore-forming clostridia? The termite-gut community is now recognized as being highly structured (Brune \& Friedrich, 2000). Are all homoacetogen species found localized to the same temporal and spatial niches within the gut? The results of the current study should help facilitate the design of quantitative mRNA, celllocalization, community fingerprinting, and other approaches to address all such questions.

\section{ACKNOWLEDGEMENTS}

This research was supported by infrastructure funds from the National Science Foundation (DBI-0107908), and a gift from the Schlumberger Foundation. We thank Rick Lovell for helpful advice and sharing data on an extensive FTHFS inventory in advance of its publication, and E. R. Leadbetter and our laboratory co-workers for their helpful comments.

\section{REFERENCES}

Bao, Q., Tian, Y., Li, W. \& 18 other authors (2002). A complete sequence of the T. tengcongensis genome. Genome Res 12, 689-700.

Boga, H. I., Ludwig, W. \& Brune, A. (2003). Sporomusa aerivorans sp. nov., an oxygen-reducing homoacetogenic bacterium from the gut of a soil-feeding termite. Int J Syst Evol Microbiol 53, 1397-1404.

Brauman, A., Kane, M. D., Labat, M. \& Breznak, J. A. (1992). Genesis of acetate and methane by gut bacteria of nutritionally diverse termites. Science 257, 1384-1387.

Breznak, J. A. (1994). Acetogenesis from carbon dioxide in termite guts. In Acetogenesis, pp. 303-330. Edited by H. L. Drake. New York: Chapman \& Hall.

Breznak, J. A. \& Leadbetter, J. R. (2002). Termite gut spirochetes. In The Prokaryotes - an Evolving Electronic Resource for the Microbiological Community. Edited by M. Dworkin, S. Falkow, E. Rosenberg, K. H. Schleifer \& E. Stackebrandt. New York: Springer. 
Breznak, J. A. \& Switzer, J. M. (1986). Acetate synthesis from $\mathrm{H}_{2}$ plus $\mathrm{CO}_{2}$ by termite gut microbes. Appl Environ Microbiol 52, 623-630.

Breznak, J. A., Switzer, J. M. \& Seitz, H. J. (1988). Sporomusa termitida sp. nov., an $\mathrm{H}_{2} / \mathrm{CO}_{2}$-utilizing acetogen isolated from termites. Arch Microbiol 150, 282-288.

Brune, A. \& Friedrich, M. (2000). Microecology of the termite gut: structure and function on a microscale. Curr Opin Microbiol 3, 263-269.

Casjens, S., Palmer, N., van Vugt, R. \& 12 other authors (2000). A bacterial genome in flux: the twelve linear and nine circular extrachromosomal DNAs in an infectious isolate of the Lyme disease spirochete Borrelia burgdorferi. Mol Microbiol 35, 490-516.

Drake, H. L., Daniel, S. L., Küsel, K., Matthies, C., Kuhner, C. \& Braus-Stromeyer, S. (1997). Acetogenic bacteria: what are the in situ consequences of their diverse metabolic versatilities? Biofactors $\mathbf{6}$, $13-24$.

Eisen, J. A., Nelson, K. E., Paulsen, I. T. \& 32 other authors (2002). The complete genome sequence of Chlorobium tepidum TLS, a photosynthetic, anaerobic, green-sulfur bacterium. Proc Natl Acad Sci U S A 99, 9509-9514.

Ferretti, J. J., McShan, W. M., Ajdic, D. \& 20 other authors (2001). Complete genome sequence of an M1 strain of Streptococcus pyogenes. Proc Natl Acad Sci U S A 98, 4658-4663.

Fraser, C. M., Norris, S. J., Weinstock, G. M. \& 30 other authors (1998). Complete genome sequence of Treponema pallidum, the syphilis spirochete. Science 281, 375-388.

Goldman, N. \& Whelan, S. (2000). Statistical tests of gammadistributed rate heterogeneity in models of sequence evolution in phylogenetics. Mol Biol Evol 17, 975-978.

Goodner, B., Hinkle, G., Gattung, S. \& 28 other authors (2001). Genome sequence of the plant pathogen and biotechnology agent Agrobacterium tumefaciens C58. Science 294, 2323-2328.

Kane, M. D. \& Breznak, J. A. (1991). Acetonema longum gen. nov. sp. nov., an $\mathrm{H}_{2} / \mathrm{CO}_{2}$ acetogenic bacterium from the termite, Pterotermes occidentis. Arch Microbiol 156, 91-98.

Kane, M. D., Brauman, A. \& Breznak, J. A. (1991). Clostridium mayombei sp. nov., an $\mathrm{H}_{2} / \mathrm{CO}_{2}$ acetogenic bacterium from the gut of the African soil-feeding termite, Cubitermes speciosus. Arch Microbiol 156, 99-104.

Kapatral, V., Anderson, I., Ivanova, N. \& 22 other authors (2002). Genome sequence and analysis of the oral bacterium Fusobacterium nucleatum strain ATCC 25586. J Bacteriol 184, 2005-2018.

Kuroda, M., Ohta, T., Uchiyama, I. \& 34 other authors (2001). Whole genome sequencing of meticillin-resistant Staphylococcus aureus. Lancet 357, 1225-1240.

Leadbetter, J. R., Schmidt, T. M., Graber, J. R. \& Breznak, J. A. (1999). Acetogenesis from $\mathrm{H}_{2}$ plus $\mathrm{CO}_{2}$ by spirochetes from termite guts. Science 283, 686-689.

Leaphart, A. B. \& Lovell, C. R. (2001). Recovery and analysis of formyltetrahydrofolate synthetase gene sequences from natural populations of acetogenic bacteria. Appl Environ Microbiol 67, 1392-1395.

Leaphart, A. B., Friez, M. J. \& Lovell, C. R. (2003). Formyltetrahydrofolate synthetase sequences from salt marsh plant roots reveal a diversity of acetogenic bacteria and other bacterial functional groups. Appl Environ Microbiol 69, 693-696.

Liesack, W., Bak, F., Kreft, J. U. \& Stackebrandt, E. (1994). Holophaga foetida gen. nov., sp. nov., a new, homoacetogenic bacterium degrading methoxylated aromatic compounds. Arch Microbiol 162, 85-90.
Lilburn, T. G., Schmidt, T. M. \& Breznak, J. A. (1999). Phylogenetic diversity of termite gut spirochaetes. Environ Microbiol 1, 331-345. Lilburn, T. G., Kim, K. S., Ostrom, N. E., Byzek, K. R., Leadbetter, J. R. \& Breznak, J. A. (2001). Nitrogen fixation by symbiotic and free-living spirochetes. Science 292, 2495-2498.

Lovell, C. R., Przybyla, A. \& Ljungdahl, L. G. (1988). Cloning and expression in Escherichia coli of the Clostridium thermoaceticum gene encoding thermostable formyltetrahydrofolate synthetase. Arch Microbiol 149, 280-285.

Ng, W.-L., Schummer, M., Cirisano, F. D., Baldwin, R. L., Karlan, B. Y. \& Hood, L. (1996). High-throughput plasmid mini preparations facilitated by micro-mixing. Nucleic Acids Res 24, 5045-5047.

Nolling, J., Breton, G., Omelchenko, M. V. \& 16 other authors (2001). Genome sequence and comparative analysis of the solventproducing bacterium Clostridium acetobutylicum. J Bacteriol $\mathbf{1 8 3}$ 4823-4838.

Odelson, D. A. \& Breznak, J. A. (1983). Volatile fatty acid production by the hindgut microbiota of xylophagous termites. Appl Environ Microbiol 45, 1602-1613.

Page, R. D. M. (1996). TREeview: an application to display phylogenetic trees on personal computers. Comput Appl Biosci 12, 357-358.

Paster, B. J., Dewhirst, F. E., Cooke, S. M., Fussing, V., Poulsen, L. K. \& Breznak, J. A. (1996). Phylogeny of not-yet-cultured spirochetes from termite guts. Appl Environ Microbiol 62, 347-352.

Purdy, K., Embley, T., Takii, S. \& Nedwell, D. (1996). Rapid extraction of DNA and rRNA from sediments by a novel hydroxyapatite spin-column method. Appl Environ Microbiol 62, 3905-3907.

Radfar, R., Shin, R., Sheldrick, G. M., Minor, W., Lovell, C. R., Odom, J. D., Dunlap, R. B. \& Lebioda, L. (2000a). The crystal structure of $N^{10}$-formyltetrahydrofolate synthetase from Moorella thermoacetica. Biochemistry 39, 3920-3926.

Radfar, R., Leaphart, A., Brewer, J. M., Minor, W., Odom, J. D., Dunlap, R. B., Lovell, C. R. \& Lebioda, L. (2000b). Cation binding and thermostability of FTHFS monovalent cation binding sites and thermostability of $N^{10}$-formyltetrahydrofolate synthetase from Moorella thermoacetica. Biochemistry 39, 14481-14486.

Ragsdale, S. W. (1997). The eastern and western branches of the Wood/Ljungdahl pathway: how the east and west were won. Biofactors 6, 3-11.

Raponi, M., Dawes, I. W. \& Arndt, G. M. (2000). Characterization of flanking sequences using long inverse PCR. Biotechniques 28, 838-844.

Ruepp, A., Graml, W., Santos-Martinez, M. L. \& 7 other authors (2000). The genome sequence of the thermoacidophilic scavenger Thermoplasma acidophilum. Nature 407, 508-513.

Schink, B., Thiemann, V., Laue, H. \& Friedrich, M. W. (2002). Desulfotignum phosphitoxidans sp. nov., a new marine sulfate reducer that oxidizes phosphite to phosphate. Arch Microbiol 177, 381-391.

Schmidt, H. A., Strimmer, K., Vingron, M. \& von Haeseler, A. (2002). TREE-PUZZLE: maximum likelihood phylogenetic analysis using quartets and parallel computing. Bioinformatics 18, 502-504.

Schöniger, M. \& von Haeseler, A. (1999). Toward assigning helical regions in alignments of ribosomal RNA and testing the appropriateness of evolutionary models. J Mol Evol 49, 691-698.

Shimizu, T., Ohtani, K., Hirakawa, H. \& 7 other authors (2002). Complete genome sequence of Clostridium perfringens, an anaerobic flesh-eater. Proc Natl Acad Sci U S A 99, 996-1001.

Tholen, A. \& Brune, A. (2000). Impact of oxygen on metabolic fluxes and in situ rates of reductive acetogenesis in the hindgut of the 
wood-feeding termite Reticulitermes flavipes. Environ Microbiol 2, $436-449$.

White, O., Eisen, J. A., Heidelberg, J. F. \& 29 other authors (1999). Genome sequence of the radioresistant bacterium Deinococcus radiodurans $\mathrm{R} 1$. Science 286, 1571-1577.
Wood, H. G. (1991). Life with $\mathrm{CO}$ or $\mathrm{CO}_{2}$ and $\mathrm{H}_{2}$ as a source of carbon and energy. FASEB J 5, 156-163.

Wood, D. W., Setubal, J. C., Kaul, R. \& 48 other authors (2001). The genome of the natural genetic engineer Agrobacterium tumefaciens C58. Science 294, 2317-2323.

Front cover illustration: A phase contrast image of diluted gut contents from the termite Zootermopsis angusticollis exhibiting a diversity of spirochaetal morphotypes. Micrograph courtesy Jared R. Leadbetter (Environmental Science \& Engineering, California Institute of Technology, Pasadena, CA, USA) and John A. Breznak (Department of Microbiology, Michigan State University, East Lansing, MI, USA). 